\title{
SIMTON: A Simulator for Transparent Optical Networks
}

\author{
Daniel A. R. Chaves, Helder A. Pereira, Carmelo J. A. Bastos-Filho and Joaquim F. Martins-Filho
}

\begin{abstract}
In this article we present a software to simulate Transparent Optical Networks (SIMTON). SIMTON is an eventdriven simulation software implemented in $\mathrm{C}++$ which takes into account optical device characteristics for the evaluation of network blocking probability in wavelength routed transparent optical networks. The simulator uses a physical layer model that considers the following effects: device losses, fiber attenuation, four wave mixing, residual chromatic dispersion and polarization mode dispersion in optical fibers, gain saturation in optical amplifiers (Erbium Doped Fiber Amplifier), dependence of the amplified spontaneous emission noise with the input power in EDFA, in-band crosstalk in optical switches, and source spontaneous emission noise of the laser transmitter. By using SIMTON it is possible to adjust the parameters of the optical devices, as well as to choose the routing and wavelength assignment algorithm. Moreover, the tool has a graphical interface. We also present some examples of network analysis results obtained from SIMTON.
\end{abstract}

Index Terms-All-Optical Networks, Impairment AwareRouting and Wavelength Assignment, Simulation Software, Wavelength Division Multiplexing.

\section{INTRODUCTION}

$\mathbf{O}$ PTICAL networks are currently the most used technology by the telecommunications carriers for the implementation of their backbones. The main reason for this is that the optical communications systems offer, at the same time, the possibility to expand the transmission capacity allied to a high availability and reliability [1], [2].

The optical networks can be classified in three categories: opaque, all-optical and translucent networks [3]. In the opaque networks, the optical layer is used only for transmission. All network operations, such as switching and management, are carried out by electronic circuits. In these networks, at each network node along the lightpath, the optical signal undergoes optical-electronic-optical $(\mathrm{O} / \mathrm{E} / \mathrm{O})$ conversions [4]. On the other hand, in all-optical networks the optical signal propagates along the network, from the source node to the destination node, in the optical domain without experiencing $\mathrm{O} / \mathrm{E} / \mathrm{O}$ conversions [4]. The translucent networks are optical networks that use the concept of islands of transparency. These networks are divided in many transparent islands and these islands are interconnected by electronic regenerators [3].

Although the all-optical networks are less expensive than the opaque ones, in these networks there is no signal regeneration

Manuscript received November 1, 2010; revised March 25, 2011.

Daniel A. R. Chaves, Helder A. Pereira and Carmelo J. A. Bastos-Filho are with Polytechnic School of Pernambuco, University of Pernambuco, 50750-470, Recife-PE, Brazil. Emails:\{darc,carmelofilho\}@ecomp.poli.br, helder.pereira@poli.br.

Joaquim F. Martins-Filho is with Photonics Group, Department of Electronics and Systems, Federal University of Pernambuco, Avenida da Arquitetura, s/n Bloco A, $4^{\circ}$ Andar, Sala 408, Cidade Universitaria, 50740-550, Recife-PE, Brazil. Tel: (81) 2126-7784. Fax: (81) 2126-8215. Email: jfmf@ufpe.br. along the lightpaths. Thus, the signal is degraded during transmission, and the quality of transmission of the lightpath can not reach acceptable levels $(e . g$. leading to a high bit error rates) [5]. Therefore, for the implementation of such networks, it becomes necessary to estimate the degradation of the optical signal along the possible lightpaths [5]. In order to estimate the impacts of signal degradation in the optical layer, three strategies can be adopted: the use of numerical calculations for wave propagation in optical fibers and devices (numerical solution), the implementation of an optical testbed (experimental solution) or the use computers to simulate the network operation. Despite being very accurate, the first one is impracticable for large networks evaluation due to the high computational complexity of the problem, specially if the target is to assess the dynamic behavior of the network. The second option is particularly expensive due to the high cost of the optical devices deployed in the networks. As a good alternative, the network designers can use computational simulations with simplified analytical models. Using these models, it is possible for a simulator to test, compare and evaluate the performance of devices, algorithms, protocols and topologies used in the network.

Following the third strategy (the simulation idea), some simulators of optical networks have been developed. These tools can be divided in two groups. In the first group, the tools do not consider the degradation of the signal in the optical layer, which means that they can only be applied to opaque networks. In this first group the following projects can be listed: OWNS [6] (extension of the Network Simulator [7]), OPNET [8], NIST Merlin [9], and TONetS [10]. In the second group, the tools take into account the degradation of the signal quality in the optical layer along the optical signal propagation process, and for this reason, they are able to simulate the behavior of transparent all-optical networks. The simulator SIMON [11] is classified in this group and considers the effects of attenuation of the optical signal in optical fibers and in other devices, gain saturation in optical amplifiers and in-band crosstalk in the optical switches.

We have recently presented a simulation tool called SIMTON [12], which considers, in addition to the effects considered by SIMON, the following optical layer impairments: polarization mode dispersion (PMD), residual chromatic dispersion (RCD), four wave mixing (FWM) and the dependence of amplified spontaneous emission noise (ASE) with the Erbium doped fiber amplifier (EDFA) input optical power [13], [14]. In this paper we present an extended version of [12] for the full description of the simulation tool, since we included the physical impairments model, additional simulation results and references.

This paper is organized as follows: in Section II we declare the SIMTON implementation assumptions; in Section III we 
present the analytical model used by SIMTON to evaluate the optical physical impairments; in Section IV we show the SIMTON graphical interface; in Section $\mathrm{V}$ we present the statistical analysis of the simulator, in Section VI we list its cases of use; in Section VII we show some examples of simulation results and, finally; in Section VIII we give our conclusion.

\section{IMPLEMENTATION ASSUMPTIONS}

The simulation tool allows one to adjust the parameters of the optical devices, as well as choosing the routing and wavelength assignment (RWA) algorithm to be used in the simulation. SIMTON was implemented in $\mathrm{C}++$ programming language and was divided in two modules: the simulator of optical network and the graphical user interface (GUI).

In this Section some aspects considered in the implementation of SIMTON are shown.

\section{A. Physical Layer}

The physical layer of the network is composed by optical devices such as optical amplifier and optical switches. Moreover, an optical network is represented by a set of nodes and a set of links interconnecting the nodes. SIMTON assumes that a pair of optical fibers is deployed in each link, one for transmission and the other for reception. Thus, each fiber has an unidirectional traffic. In SIMTON, each connection is established in a bidirectional way. Despite we have assumed this, it is easy to set an unique fiber for transmission and reception.

This first version of SIMTON does not support the wavelength conversion capability in the nodes along the ligthpaths. Therefore, each connection is carried out in the same wavelength from the source to the destination node. SIMTON uses an optical circuit switching approach. The optical amplifiers gains can be easily adjusted to exactly compensate for the total link losses.

The link architecture and optical devices assumed by SIMTON are shown in Fig. 1. From the left to the right we have the following devices: laser transmitter, optical switch, optical multiplexer, optical fiber, optical demultiplexer, optical switch and optical receptor. Each device has a set of parameters that can be adjusted by the SIMTON user. The set of parameters for each network device is listed in Table I, which also shows the standard preset values used in the simulations described in Section V and in Section VII.

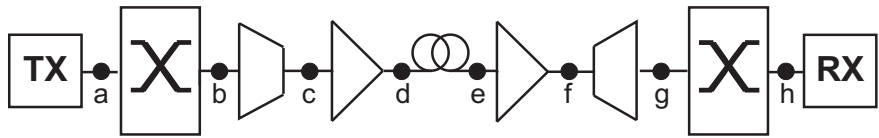

Fig. 1. The link architecture and optical devices assumed by SIMTON.

In order to take into account the physical impairments the SIMTON uses a physical layer model developed by our research group [13], which evaluates the optical signal-to-noise ratio (OSNR) of the lightpath. This evaluation is based on the analytical model shown in Section III. The verification of the quality of signal (QoS) of one lightpath is made by comparing the $O S N R_{\text {out }}$ in the output of the lightpath with the predefined
TABLE I

LIST OF THE OPTICAL DEVICES AND PARAMETERS ADJUSTABLE BY THE SIMTON USER.

\begin{tabular}{|c|c|c|}
\hline Device & Parameter & Standard value \\
\hline \multirow[t]{3}{*}{ "Network } & Load & 70 Erlang \\
\hline & Maximum temporal broadening & $10 \%$ \\
\hline & $\begin{array}{l}\text { Minimum acceptable signal-to- } \\
\text { noise ratio }\end{array}$ & $23 \mathrm{~dB}$ \\
\hline \multirow{5}{*}{$\begin{array}{l}\text { Laser } \\
\text { transmitter }\end{array}$} & First used wavelength & $1528.78 \mathrm{~nm}$ \\
\hline & Laser power & $0 \mathrm{dBm}$ \\
\hline & Linewidth & $0.0045 \mathrm{~nm}$ \\
\hline & Output signal-to-noise ratio & $30 \mathrm{~dB}$ \\
\hline & Transmission bit rate & $40 \mathrm{Gbps}$ \\
\hline \multirow{2}{*}{$\begin{array}{l}\text { Optical } \\
\text { switch }\end{array}$} & Insertion loss & $3 \mathrm{~dB}$ \\
\hline & Isolation factor & $-40 \mathrm{~dB}$ \\
\hline Multiplexer & Insertion loss & $3 \mathrm{~dB}$ \\
\hline \multirow{3}{*}{$\begin{array}{l}\text { Optical } \\
\text { amplifier }\end{array}$} & Noise figure & $5 \mathrm{~dB}$ \\
\hline & Non-saturated gain & Compensate for \\
\hline & Output saturation power & $19 \mathrm{dBm}$ \\
\hline \multirow{10}{*}{$\begin{array}{l}\text { Optical } \\
\text { fiber }\end{array}$} & Channel spacing & $100 \mathrm{GHz}$ \\
\hline & Loss coefficient & $0.22 \mathrm{~dB} / \mathrm{km}$ \\
\hline & Number of wavelengths per link & \\
\hline & $\begin{array}{l}\text { Transmission fiber dispersion } \\
\text { coefficient (@1544.53 nm) }\end{array}$ & $-0.75 \mathrm{ps} / \mathrm{km} . \mathrm{nm}$ \\
\hline & $\begin{array}{lll}\text { Transmission } & \text { fiber } & \text { slope } \\
(@ 1544.53 \mathrm{~nm}) & & \end{array}$ & $0.06 \mathrm{ps} / \mathrm{km} . \mathrm{nm}^{2}$ \\
\hline & $\begin{array}{l}\text { Zero dispersion wavelength for } \\
\text { transmission fiber }\end{array}$ & $1557 \mathrm{~nm}$ \\
\hline & $\begin{array}{l}\text { Compensating fiber dispersion } \\
\text { coefficient (@1544.53 nm) }\end{array}$ & $-99.77 \mathrm{ps} / \mathrm{km} . \mathrm{nm}$ \\
\hline & $\begin{array}{lll}\begin{array}{l}\text { Compensating } \\
(@ 1544.53 \mathrm{~nm})\end{array} & \text { fiber } & \text { slope }\end{array}$ & $-1.87 \mathrm{ps} / \mathrm{km} \cdot \mathrm{nm}^{2}$ \\
\hline & $\begin{array}{l}\text { Zero residual dispersion } \\
\text { wavelength }\end{array}$ & $1544.53 \mathrm{~nm}$ \\
\hline & PMD coefficient for NZ-DSF & $0.04 \mathrm{ps} / \sqrt{\mathrm{km}}$ \\
\hline
\end{tabular}

minimum OSNR $\left(O S N R_{\mathrm{Th}}\right)$. Thus, in order to satisfy the QoS requirement, the $O S N R_{\text {out }}$ of the lightpath must be greater than $O S N R_{\mathrm{Th}}$.

\section{B. Routing and Wavelength Assignment Module}

Our network simulation engine follows the flowchart shown in Fig. 2. A candidate lightpath for the incoming call request is searched by the RWA module. The output of the RWA module feeds the call admission control (CAC) module. The CAC is responsible for the decision whether a given call request can be established or not in the network. If the RWA module can not find a lightpath for a requested call, the CAC blocks it.

If the RWA algorithm returns a candidate lightpath (CLP), then the CAC evaluates its $\Delta t_{\mathrm{CLP}}$ and $O S N R_{\mathrm{CLP}}$ using the physical layer impairments (PLI) model (Section III). The current state of the network is considered in this evaluation. The CAC checks if both QoS constraints are satisfied: $\Delta t_{\text {CLP }}$ $\leq \Delta t_{\mathrm{Th}}$ and $O S N R_{\mathrm{CLP}} \geq O S N R_{\mathrm{Th}}$, where $\Delta t_{\mathrm{Th}}$ is the maximum pulse broadening and $O S N R_{\mathrm{Th}}$ is the minimum acceptable OSNR value for the QoS requirements. If either inequality does not hold, then the requested call is blocked by the CAC. Otherwise, the request is accepted. An accepted call results in the establishment of a circuit switched bidirectional connection in two different fibers between the selected source and destination nodes. 


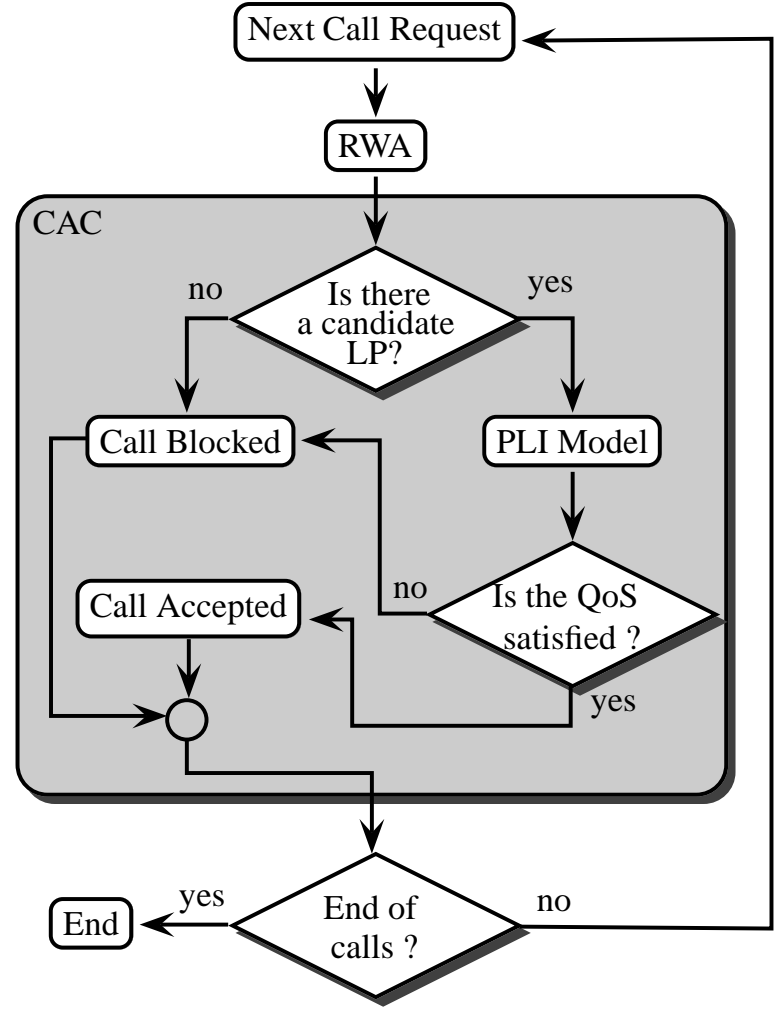

Fig. 2. Flowchart used by SIMTON for the solution of the RWA problem.

\section{Traffic Generation and Network Load}

To simulate the dynamic behavior of the network, the call requests are generated dynamically following a stochastic process. This approach is also known as dynamic lightpath establishment (DLE) [15]. For each call request, two network nodes are raffled, randomly, following an uniform probability density function. Thus, all the nodes in the network are equally probable to be selected. These two selected nodes are assigned as the source and destination nodes of the call. The generation process of call requests follows a Poisson process: the time interval between calls is exponentially distributed with average $\mu$ and the duration of each call also follows an exponential distribution with a mean value equal to $\frac{1}{H}$. With these two parameters, one can define the network load $L$ as $L=\mu H$, in which, $H$ is the mean time, on average, that the call is active and $\mu$ is the mean rate for the call requests generation. The network load $L$ is given in Erlang.

\section{Blocking Probability Evaluation}

The network blocking probability estimates the relative amount of not accepted calls by the network. As it was discussed before, a blocked call can occur either by the lack of available network resources to establish the call or for inadequate quality of signal for the found route. Thus, a higher blocking probability means that a larger number of users could not make use of the network resources, which indicates a worse network performance. The blocking probability is estimated by evaluating the ratio between the number of blocked calls and the total number of call requests to the network.

\section{Physical Layer Modeling}

The physical layer impairments model used by SIMTON is described in details in [13]. However, other physical impairment models, available in the literature, can be implemented in the SIMTON simulation tool for transparent optical network performance analysis. A recentt review on physical layer models can be found in [16]. Here we present a summary concerning the physical impairments considered in this paper. The formulation quantifies the OSNR degradation along the optical signal propagation in the all-optical networks. The impact of physical layer impairments is taken into account by considering the signal power and the noise power at the destination node, both affected by gains and losses along the lightpath. Moreover, some network elements add noise components or have a nonlinear response. The optical amplifiers add ASE noise power and are also affected by gain saturation and ASE depletion as the total input optical signal power increases. The optical switches add noise due to non-ideal isolation between ports. And the transmission fibers add noise due to the FWM effect when the signal wavelength is close to zero dispersion wavelength $\left(\lambda_{0}\right)$ and also induce pulse broadening due to PMD and RCD. Fig. 3 shows the configuration of the network wavelength routing node. It is similar to the one assumed in [11].

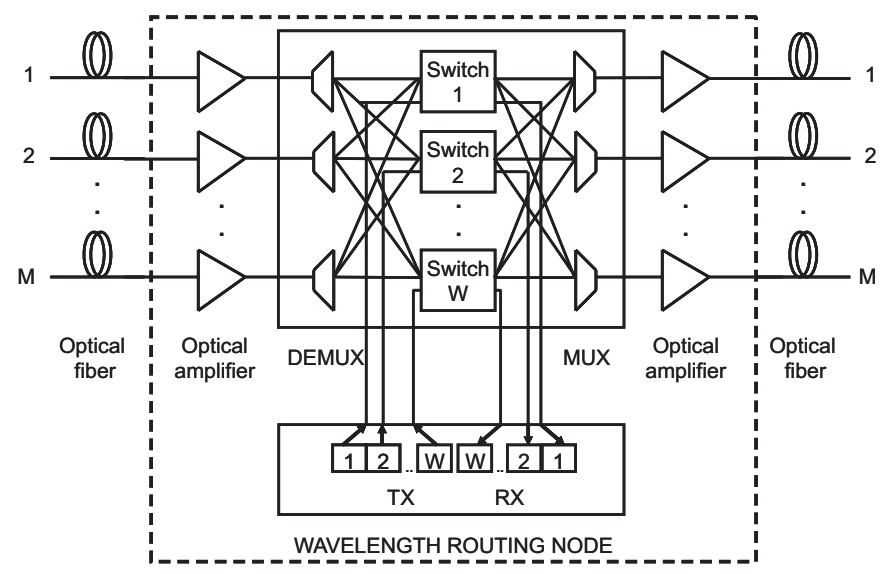

Fig. 3. Network wavelength routing node configuration, for $M$ fibers with $W$ wavelengths.

Fig. 1 shows the network devices considered by the model in each link. The points $\mathbf{a}$ to $\mathbf{h}$ are evaluation points where the signal and noise can be determined in the optical domain. At point a, we have the input optical signal power $\left(P_{\text {in }}\right)$ and the input optical noise power $\left(N_{\text {in }}\right)$. The ratio between $P_{\text {in }}$ and $N_{\text {in }}$ defines the OSNR of the transmitter $\left(O S N R_{\text {in }}\right)$. For the lightpath with $i$ links, the elements between $\mathbf{b}$ and $\mathbf{h}$ are repeated $(i-1)$ times before the signal reaches the receiver at the destination node. At points $\mathbf{b}$ and $\mathbf{h}$ in Fig. 1, we considered the noise induced by in-band crosstalk in the optical switches assuming that the out-band crosstalk can be filtered out on the switch output. At points $\mathbf{c}$ and $\mathbf{g}$, we considered the contribution of the multiplexer and demultiplexer elements. Brandt-Pearce et al [17] considered a source of crosstalk due to imperfect WDM demultiplexing. They found that it is significant for high density of channels in the network. However, in this paper we only took into account the multiplexer and demultiplexer losses. At points $\mathbf{d}$ and $\mathbf{f}$, the noise induced by the optical amplifiers, as 
well as the gain saturation effect, was considered. At point $\mathbf{e}$, we considered the noise generated by the FWM effect. This nonlinear effect depends on the channel spacing, the optical signal power per channel, the number of wavelengths propagating in the optical fiber, the fiber dispersion coefficient and the zero dispersion wavelength of the transmission fiber [18]. Finally, at the point $\mathbf{h}$, one can evaluate the output optical signal power $\left(P_{\text {out }}\right)$ and the output optical noise power $\left(N_{\text {out }}\right)$.

$P_{\text {out }}$ is evaluated according to the gains and losses along the optical signal propagation and it is given by:

$$
P_{\text {out }}=\frac{G_{\mathrm{Amp}_{1}} e^{-\alpha d} G_{\mathrm{Amp}_{2}}}{L_{\mathrm{SW}}^{2} L_{\mathrm{Mx}} L_{\mathrm{Dx}}} P_{\mathrm{in}},
$$

where $G_{\mathrm{Amp}_{1}}$ and $G_{\mathrm{Amp}_{2}}$ are the dynamic linear gains of the booster and pre-amplifier respectively. $\alpha$ is the fiber loss coefficient and $d$ is the fiber length. $L_{\mathrm{Sw}}, L_{\mathrm{Mx}}$ and $L_{\mathrm{Dx}}$ are the optical switch, multiplexer and demultiplexer losses, respectively.

$N_{\text {out }}$ is evaluated from the source node to the destination node, including the noise components in the respective points of evaluation (a to $\mathbf{h}$ in Fig. 1) along the lightpath and it is given by:

$$
\begin{aligned}
N_{\mathrm{out}}= & \frac{G_{\mathrm{Amp}_{1}} e^{-\alpha d} G_{\mathrm{Amp}_{2}}}{L_{\mathrm{Mx}} L_{\mathrm{Dx}} L_{\mathrm{Sw}}^{2}} N_{\mathrm{in}}+ \\
& \frac{G_{\mathrm{Amp}_{1}} e^{-\alpha d} G_{\mathrm{Amp}_{2}}}{L_{\mathrm{Mx}} L_{\mathrm{Dx}} L_{\mathrm{Sw}}} \varepsilon \sum_{j=1}^{n} P_{\mathrm{Sw}_{1, j}}(\lambda)+ \\
& +\frac{G_{\mathrm{Amp}_{2}}}{L_{\mathrm{Dx}} L_{\mathrm{Sw}}} \sum_{j=1}^{m} P_{\mathrm{FWM}_{j}}(\lambda)+\varepsilon \sum_{j=1}^{s} P_{\mathrm{Sw}_{2, j}}(\lambda)+ \\
& +\frac{G_{\mathrm{Amp}_{1}} e^{-\alpha d} G_{\mathrm{Amp}_{2}} \frac{h \nu(\lambda) B_{0}}{L_{\mathrm{Dx}} L_{\mathrm{Sw}_{1}}}}{2} \\
& \left(F_{\mathrm{Amp}_{1}}+\frac{F_{\mathrm{Amp}_{2}}}{e^{-\alpha d} G_{\mathrm{Amp}_{1}}}\right),
\end{aligned}
$$

where $N_{\text {in }}$ is the noise power at the transmitter output, i.e. the laser SSE, $P_{\mathrm{Sw}_{k, j}}(\lambda)$ is the optical power from the $k$-th optical switch in the $j$-th optical fiber in the same wavelength of the reference optical signal, $\varepsilon$ is the switch isolation factor and $n$ is the number of signals in the same wavelength at the switch input ports. For the node configuration used here, shown in Fig. 3, $n=M$. Pointurier et al [19] considered that $\varepsilon$ has a dynamic effect depending on the network status, whereas in [20] the authors considered that fiber nonlinearities enhance the detuned crosstalk when signals are transmitted over long distances. For the sake of simplicity, we considered here that $\varepsilon$ is the same for every wavelength, $e . g$. it does not depend on the wavelength. $P_{\mathrm{FWM}_{j}}(\lambda)$ is one of the $m$ optical power components generated by the FWM effect that falls into the same propagating signal wavelength, $h$ is the Planck constant, $\nu$ is the optical signal frequency, $B_{0}$ is the optical filter bandwidth, $G_{\text {Amp }}$ is the linear dynamic amplifier gain and $F_{\text {Amp }}$ is the amplifier noise factor.

Dividing $P_{\text {out }}$ (Eq. 1) by $N_{\text {out }}$ (Eq. 2), one can obtain the OSNR at destination node $\left(O S N R_{\text {out }}\right)$. Considering a route with $i$ links, we have:

$$
P_{\mathrm{out}_{i}}=\left(\frac{G_{\mathrm{Amp}_{i, 1}} e^{-\alpha d_{i}} G_{\mathrm{Amp}_{i, 2}}}{L_{\mathrm{Mx}} L_{\mathrm{Dx}} L_{\mathrm{Sw}}}\right) P_{\text {out }_{i-1}}
$$

and

$$
\begin{aligned}
& N_{\mathrm{out}_{i}}= \\
& \frac{G_{\mathrm{Amp}_{i, 1}} e^{-\alpha d_{i}} G_{\mathrm{Amp}_{i, 2}}}{L_{\mathrm{Mx}} L_{\mathrm{Dx}} L_{\mathrm{Sw}}} N_{\mathrm{out}_{i-1}}+\frac{G_{\mathrm{Amp}_{i, 2}}}{L_{\mathrm{Dx}} L_{\mathrm{Sw}}} \sum_{j=1}^{m} P_{\mathrm{FWM}_{i, j}}(\lambda)+ \\
& \varepsilon \sum_{j=1}^{s} P_{\mathrm{Sw}_{i+1, j}}(\lambda)+\frac{G_{\mathrm{Amp}_{i, 1}} e^{-\alpha d_{i}} G_{\mathrm{Amp}_{i, 2}}}{L_{\mathrm{Dx}} L_{\mathrm{Sw}}} \frac{h \nu(\lambda) B_{0}}{2} \\
& \left(F_{\mathrm{Amp}_{i, 1}}+\frac{F_{\mathrm{Amp}_{i, 2}}}{e^{-\alpha d_{i}} G_{\mathrm{Amp}_{i, 1}}}\right)
\end{aligned}
$$

where $P_{\text {out }_{0}}=\frac{P_{\text {in }}}{L_{\mathrm{Sw}}}$ and $N_{\text {out }_{0}}=\frac{N_{\text {in }}}{L_{\mathrm{Sw}}}+\varepsilon \sum_{j=1}^{n} P_{\mathrm{Sw}_{1, j}}(\lambda)$. Furthermore, we also considered the pulse broadening effect caused by PMD in a route using the following expression [5]:

$$
\Delta t_{\mathrm{PMD}}=\sqrt{\sum_{j=1}^{i} D_{\mathrm{PMD}}^{2}(j) d(j)},
$$

where $B$ is the transmission bit rate, $D_{\mathrm{PMD}}(j)$ is the PMD coefficient, and $d(j)$ is the length of the $j$-th link belonging to the lightpath.

The broadening of the optical pulses due to the residual chromatic dispersion is taken into account as follows [21]:

$$
\begin{gathered}
\Delta t_{\mathrm{RCD}}=\Delta \lambda_{\mathrm{Tx}} \sum_{j=i}^{i}\left\{\left[D_{\mathrm{TF}}^{\lambda_{\text {ref }}}+\left(\Delta \lambda_{j} S_{\mathrm{TF}}\right)\right] L_{\mathrm{TF}_{j}}+\right. \\
\left.\left[D_{\mathrm{DCF}}^{\lambda_{\mathrm{ref}}}+\left(\Delta \lambda_{j} S_{\mathrm{DCF}}\right)\right] L_{\mathrm{DCF}_{j}}\right\},
\end{gathered}
$$

where

$$
L_{\mathrm{DCF}_{j}}=\left|\frac{L_{\mathrm{TF}_{j}} D_{\mathrm{TF}}^{\lambda_{\text {ref }}}}{D_{\mathrm{DCF}}^{\lambda_{\text {ref }}}}\right| .
$$

$\Delta \lambda_{\mathrm{Tx}}$ represents the transmitter linewidth. $D_{\mathrm{TF}}^{\lambda_{\text {ref }}}, S_{\mathrm{TF}}$ and $L_{\mathrm{TF}_{j}}$ are the chromatic dispersion coefficient in the reference wavelength, the chromatic dispersion slope and the optical fiber length, respectively. $\Delta \lambda_{j}$ is the difference between the transmitter wavelength and the wavelength where the residual chromatic dispersion is zero.

$\delta_{\%}$ represents the temporal broadening to the optical pulse, in percentage. It can be expressed by

$$
\delta_{\%}=100 \cdot B \cdot\left(\Delta t_{\mathrm{PMD}}+\Delta t_{\mathrm{RCD}}\right),
$$

where $B$ represents the transmission bit rate. The maximum $\delta_{\%}$ is given in Table $\mathrm{I}$.

\section{Simulator ImPlEMENTATION}

Fig. 4 shows the main screen of the developed graphical interface (GUI). By using this SIMTON interface one can create and save a new network topology, or edit a saved network.

The user is allowed to configure all nodes, all links or an specific node or link parameter by selecting the desired element. Link lengths and node numbers are shown in Fig. 4. In each link, 


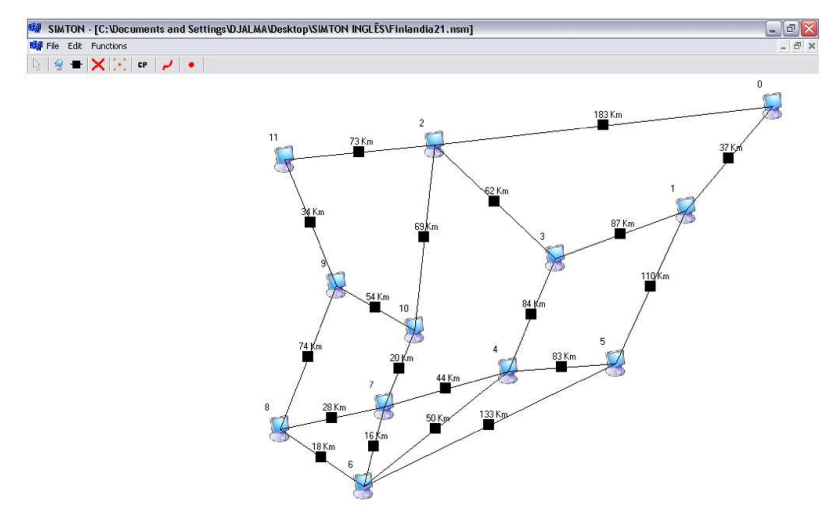

Fig. 4. SIMTON main screen showing the network topology being edited by the user.

the following parameters can be modified: optical fiber length and loss coefficient, insertion losses in the optical multiplexers and demultiplexers, number of fibers in a link, optical amplifiers gain, saturation power and noise figure. All these options are illustrated in the parameters configuration screen shown in the Fig. 5(a). In each node the following configuration options are available: switch insertion loss, optical signal-to-noise ratio of the transmitter and laser output power as illustrated in Fig. 5(b). SIMTON also allows the user to configure all amplifier gains to compensate for the losses or to set each EDFA gain of the network.

Fig. 6 illustrates the screen where the user can define the simulation parameters. Some examples are: the number of generated calls, the value for $O S N R_{\mathrm{Th}}$, which impairments should be considered or not during the simulation, the RWA algorithm, types and range of parameters in the considered analysis. The simulation process starts upon clicking the "Simulate" button.

During the simulation process, the SIMTON shows a screen indicating the simulation evolution, which is shown in Fig. 7(a). Upon the end of the simulation, the SIMTON shows a screen with the result, which is shown in Fig. 7(b). This screen always shows the blocking probability as function of the optical devices parameters or network load. In addition, the software also generates a log text file in which all the data about the simulation are recorded. Thus, it is possible to export the simulation results to another plotting software.

\section{Statistical Analysis of the Simulator}

The main result generated by SIMTON is the network blocking probability as a function of some parameters as discussed in the previous sections. In order to verify the reliability of the results given by SIMTON we performed a statistical analysis of the results. The blocking probability returned by SIMTON is a random variable that is obtained as the ratio between the number of blocked calls and the total number of call requests to the network. The simulation software generates a finite set of call requests. It is expected that the variance of the blocking probability results decreases as the number of call requests increases. In order to analyze the statistical behavior of the blocking probability results, we performed a set of 30

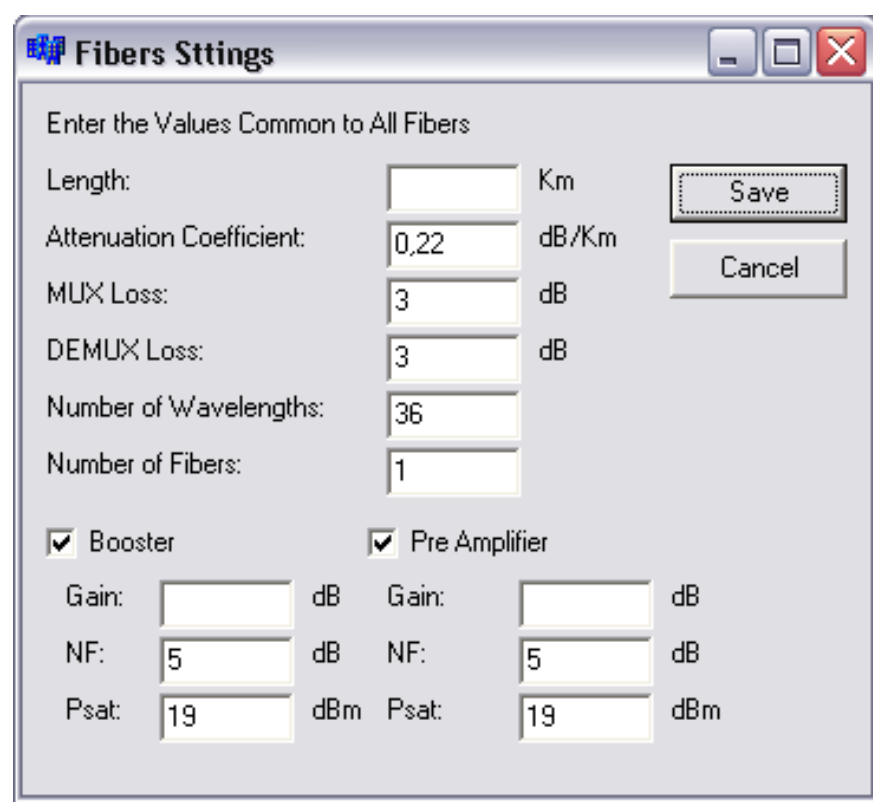

(a)

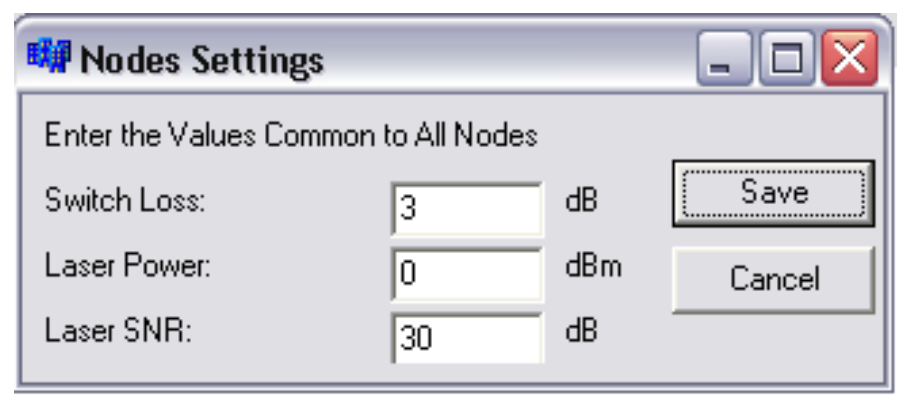

(b)

Fig. 5. Configuration screens of the optical devices parameters in network: (a) link and (b) node.

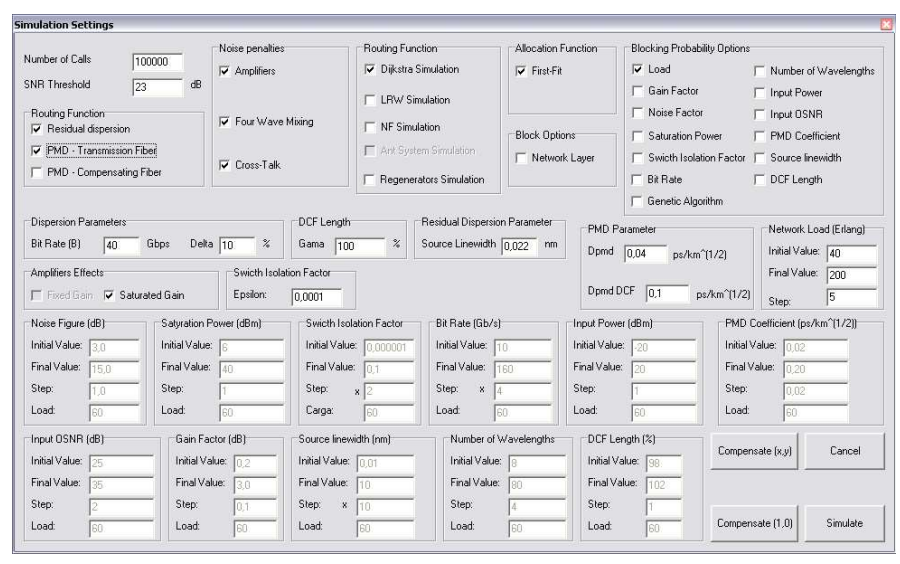

Fig. 6. Simulation setup screen - configuring the simulation parameters.

simulations using the parameters listed in Table I with a network load of 70 Erlangs in the Finland topology [22], which is shown in Fig. 4.

Fig. 8(a) shows the box plot of these results as a function of the number of call requests. The open square and the horizontal line inside the box represent the mean and the median of the results obtained from the 30 simulations, respectively. The box stands for the $25^{\text {th }}$ and $75^{\text {th }}$ percentile, whereas the whisker 


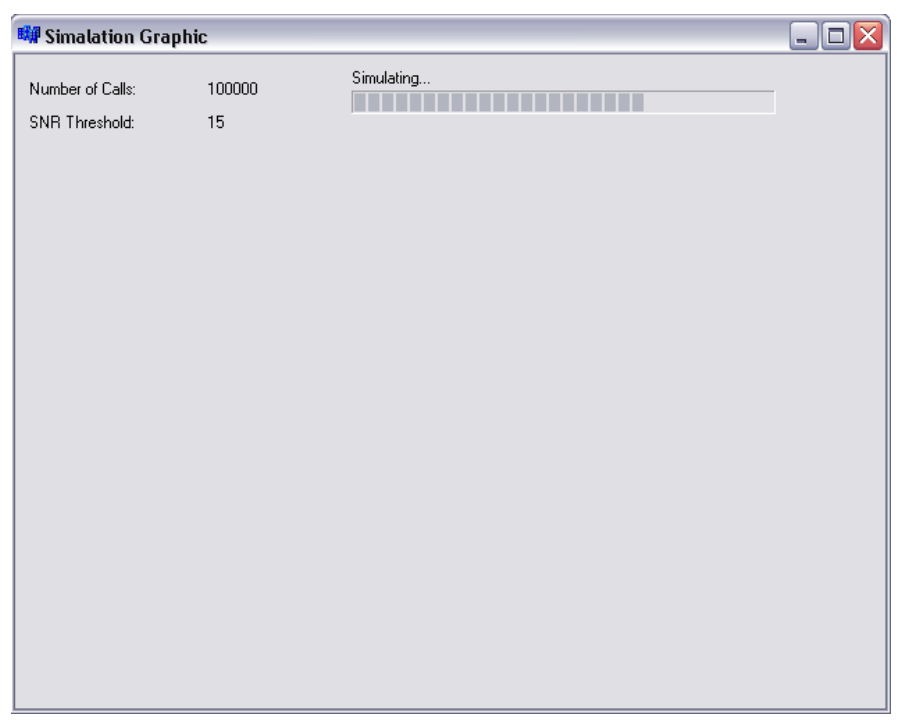

(a)

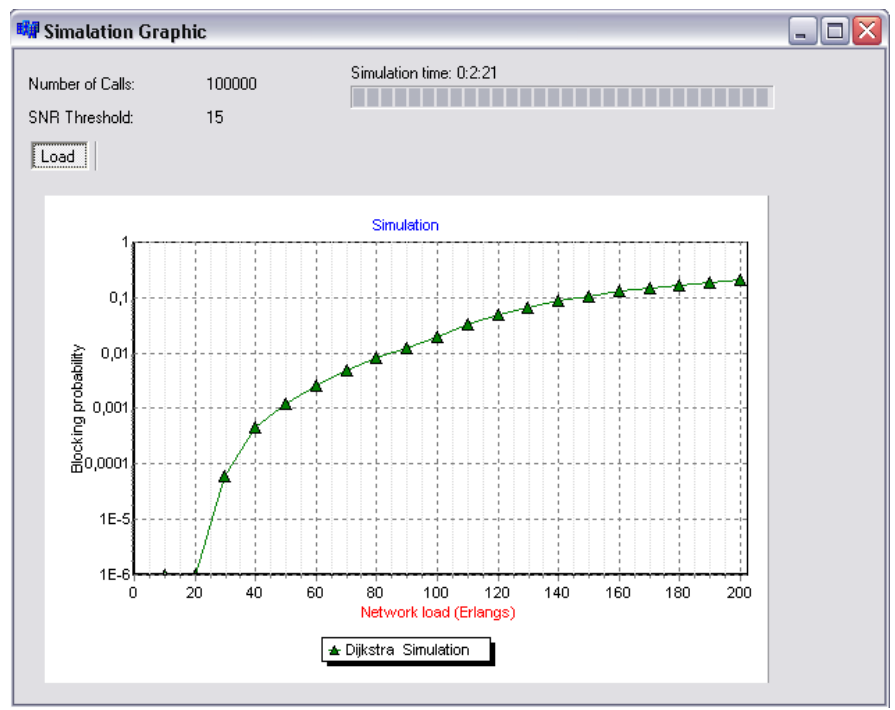

(b)

Fig. 7. Simulation screens: (a) simulating and (b) showing results.

stands for the $1^{\text {st }}$ and $99^{\text {th }}$ percentile, which means that the region in the whiskers delimitates the confidence interval of $98 \%$. As one can note from Fig. 8(a), as the number of simulated call requests increases the confidence interval of $98 \%$ becomes narrower, as expected. The width of the whiskers and the boxes obtained for $1,5 \times 10^{5}$ and $3 \times 10^{5}$ call requests are almost the same. It indicates that for this level of blocking probability $\left(8 \times 10^{-3}\right)$ a number of call requests higher than $3 \times 10^{5}$ will not lead to a narrower $98 \%$ confidence interval.

Fig. 8(b) shows the simulation time of the SIMTON, considering different scenarios of simulation. For every scenario

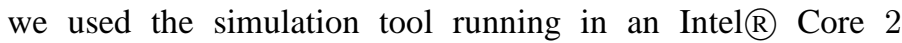
Duo @ $2.13 \mathrm{GHz}$ with $3 \mathrm{~GB}$ of RAM computer. We performed a set of 30 simulations using the parameters listed in Table I, considering a set of $10^{5}$ call requests in the Finland topology [22], which is presented in Fig. 4. Table II describes the physical impairments considered in each scenario.

In the scenarios $S 1$ to $S 4$ the FWM effect is not considered. Note from the Fig. 8(b) that, for these scenarios, the time spent

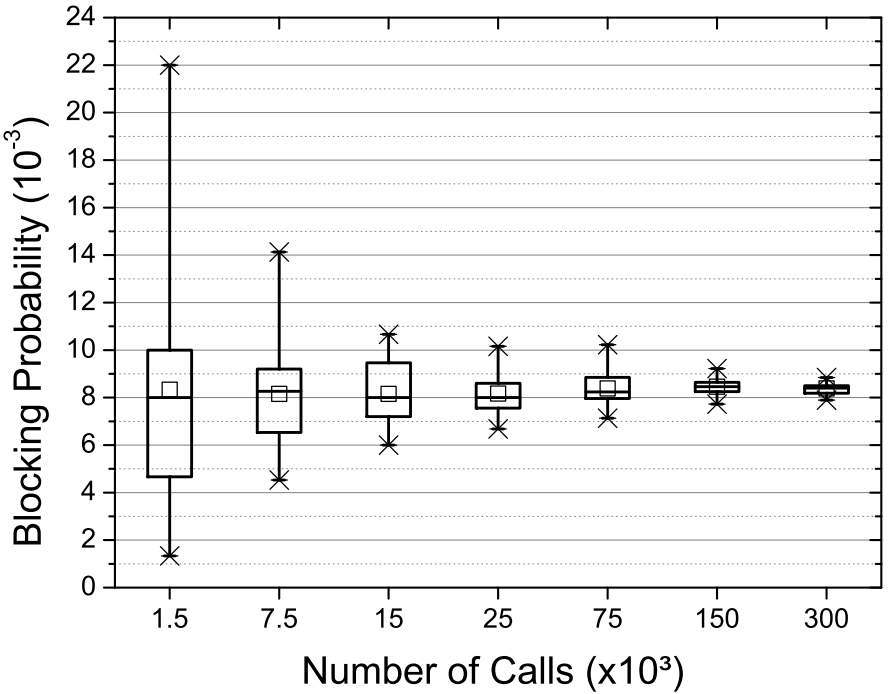

(a)

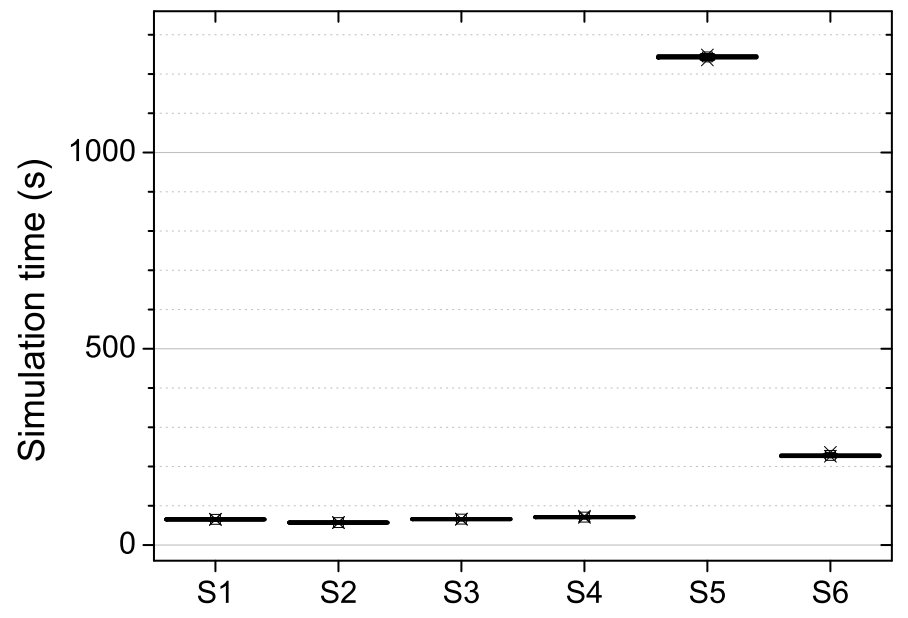

(b)

Fig. 8. Statistical analysis of the simulator: (a) convergence aspects and (b) simulation time for different scenarios.

TABLE II

DESCRIPTION OF DIFFERENT SCENARIOS CONSIDERED.

\begin{tabular}{c|l}
\hline Scenario & \multicolumn{1}{c}{ Impairment } \\
\hline \hline$S 1$ & PMD effect only. \\
\hline$S 2$ & PMD and RCD effects only. \\
\hline$S 3$ & Amplifier impairments only. \\
\hline$S 4$ & Amplifier and optical switch impairments only. \\
\hline$S 5$ & Amplifier, optical swith and FWM effect only. \\
\hline$S 6$ & All physical impairments. \\
\hline
\end{tabular}

by the SIMTON to perform the simulations are practically in the same order of magnitude (around $80 \mathrm{~s}$ ). When the FWM effect is also considered (S5), the simulation time increases by a factor of almost 16 times (around $1270 \mathrm{~s}$ ), compared to the other scenarios. It allows one to conclude that the evaluation of the FWM effect is very time consuming, compared with the other physical impairments. In $S 6$, all impairments are considered including the FWM. The main difference between the scenarios $S 5$ and $S 6$ is that the pulse broadening impairment is taken into account in $S 6$, whereas it is not in $S 5$. Note from Fig. 8(b) 
that the simulation time for the $S 5$ is considerably higher than for the $S 6$ scenario. It can be explained by the implementation of the CAC module (described in details in Section II). The $\mathrm{CAC}$ verifies the pulse broadening impairments before the other physical impairments. For this reason, when a call does not meet the pulse broadening criterion it is blocked without any further evaluation of the other physical impairments, reducing the total simulation time.

\section{SIMTON CASES OF USE}

In order to illustrate how SIMTON works, this section presents a brief description of the software cases of use. SIMTON is divided in two modules: the optical network simulator and the graphical user interface. Fig. 9(a) shows the cases of use diagram of the graphical user interface. The options available to the user are:

- Create network: Allows the user to design a new network topology.

- Save network: Allows the user to save a designed network into a file.

- Open network: Allows the user to open a previously saved network.

- Modify network: The user can edit a network by adding or removing links or nodes of the active network topology.

- Compensate for the losses: Setup the optical amplifiers gains to compensate for the losses along the links.

- Configure the devices: Allows the user to configure the parameters of network devices. There are two options to configure the devices:

- All devices: Changes simultaneously the values of the parameters for all the devices in the network.

- Single devices: Changes the value of a specific device parameter.

- Run a simulation: Starts a simulation with the parameters configured by the user.

- RWA algorithm: Allows the user to choose the RWA algorithm used during the simulation. There are four possible choices implemented for the routing algorithm: shortest path (SP) [15], least resistance weigth (LRW) [23], optical signal-to-noise ratio (OSNR-R) [13] and minimum number of hops $(\mathrm{MH})$ [15]. The wavelength assignment algorithms implemented are: first fit, random, most used, least used, best fit and just enough [15]. Other RWA algorithms can be easily implemented.

- Select results: SIMTON gives, as a result, the graph of the network blocking probability as a function of some predefined parameters. The following parameters can be chosen by the user: number of wavelengths per fiber, amplifier saturation power, amplifier noise figure, laser transmitter power, network load, switch isolation factor, transmission fiber PMD coefficient and the output OSNR of the transmitter laser.

- Select impairments: Allows the user to select which impairments SIMTON should take into account during the simulation. The possible choices are: PMD, RCD, FWM, dependence of ASE with the EDFA input power, EDFA gain saturation and switch crosstalk.

- Show graph: Plots the simulation results in a graph of blocking probability as a function of devices parameters or network load. The devices parameters choices are: amplifier noise figure, amplifier saturation power, transmission laser power, switch isolation factor, number of wavelengths, network load and PMD coefficient of the fiber, for example.

Fig. 9(b) shows the cases of use diagram of the simulation module. The options available to the user are:

- Simulate: Starts a network simulation.

- Connection request generator: Simulates the call requisition patterns in the network, according to the description in Section II-C.

- Evaluate the OSNR: Evaluates the output OSNR of the lightpath, according to Eq. (3) and Eq. (4) presented in Section III and the set of impairments chosen by the user.

- Call manager: This module handles the connections in the network, setting up connection and removing inactive calls, releasing its resources for new assignments.

- Establishment of the calls: Establishes the calls in the network and reserves network resources for the calls. Moreover, it evaluates and stores the optical powers in each network point.

- Release calls: Releases the network resources used by the ended connections.

- RWA algorithm: Runs the routing and wavelength assignment algorithm.

\section{EXAMPLES OF Simulation RESUlts}

By using the SIMTON, it is possible to perform a variety of studies concerning to the network performance (in terms of blocking probability) and to make performance comparisons of networks using different optical devices characteristics. As an example, some of the possible studies and analysis that can be performed using SIMTON are shown in this section. The detailed discussion about the simulation results presented here and their implications on network performance are not in the scope of this paper and it can be found in [13].

Fig. 10(a) shows the network performance (blocking probability) as a function of device parameters, for different network loads. It indicates that there is an optimal value for the laser power, since for low powers the blocking probability is high due to amplifier noise, and for high powers the blocking probability increases due to the non-linear effect, in-band crosstalk and amplifier saturation effects [13]. This simulation could be used by a network designer to specify the device characteristics for the optimization of network operation. For example, Fig. 10(b) shows the impact of different amplifier saturation powers and noise figures, Fig. 10(c) shows the impact of the use of dispersion shifted fibers (DSF) and non-zero DSF (NZ-DSF) and Fig. 10(d) shows the impact of optical switches with different isolation factor values on network performance.

It is also possible to analyze the impact of different PMD coefficients (Fig. 11(a)), different number of wavelengths per link (Fig. 11(b)), different transmitter bit rates (Fig. 11(c)) and source linewidths (Fig. 11(d)) on the network performance.

Besides the analysis of network performance as a function of device characteristics, SIMTON is also able to analyze and compare the network performance for different RWA 


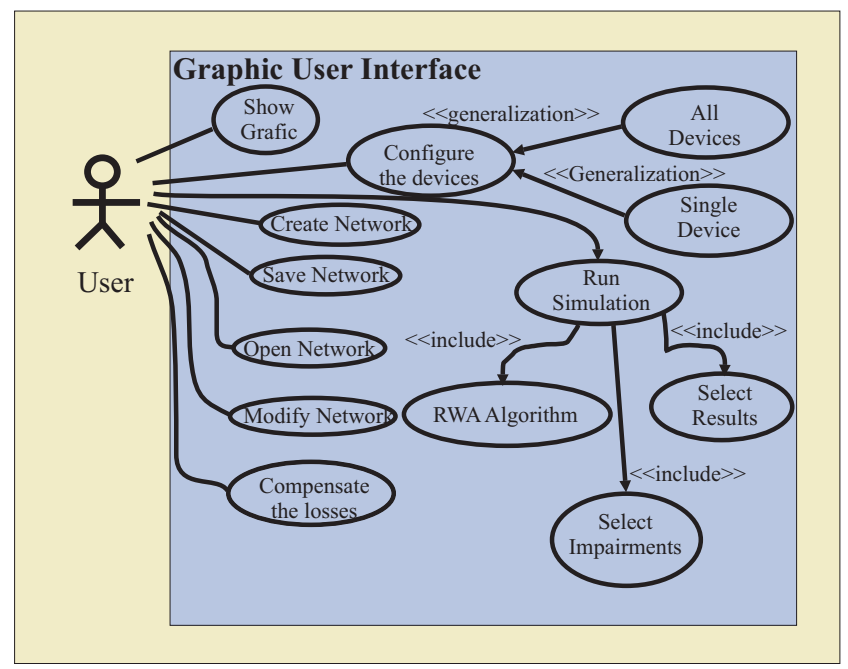

(a)

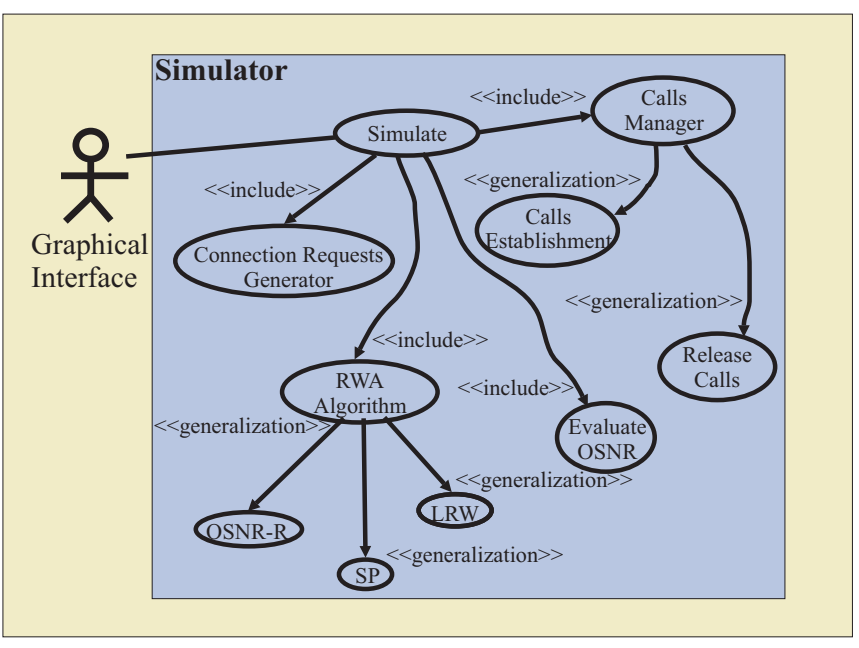

(b)

Fig. 9. Cases of use diagram for the SIMTON: (a) GUI module and (b) simulation module.

algorithms. As an example, Fig. 12 shows the network blocking probability as a function of network load for three different RWA algorithms [13], which are: SP, LRW and OSNR-R.

\section{CONCLUSIONS}

This paper presented a computational simulation tool named SIMTON designed for the simulation of wavelength routed optical networks. SIMTON can be used to investigate the impact of physical impairments in the performance of alloptical networks. SIMTON takes into account more physical impairments than the other similar simulation tools found in the literature.

We believe the SIMTON is a powerful tool for optical network simulations, to evaluate the network performance for different RWA algorithms, different device specifications, different network topologies and traffic loads. The possible users include network designers and operators, equipment vendors and network researchers, to perform network analysis, update and planning.

Although this version of SIMTON takes into account many important physical layer impairments, the authors intend to

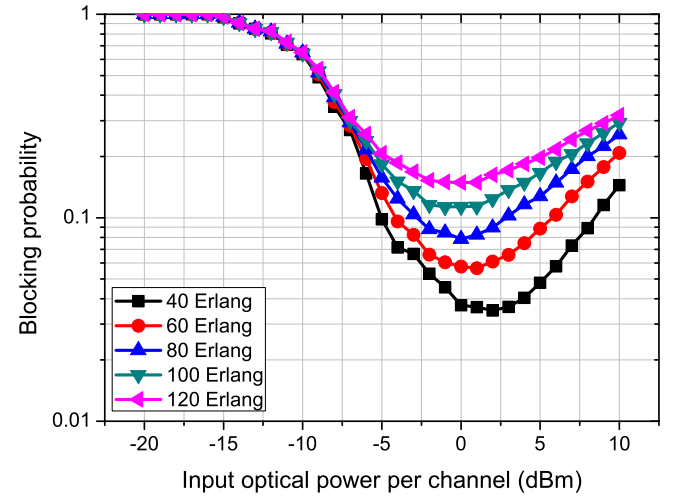

(a)

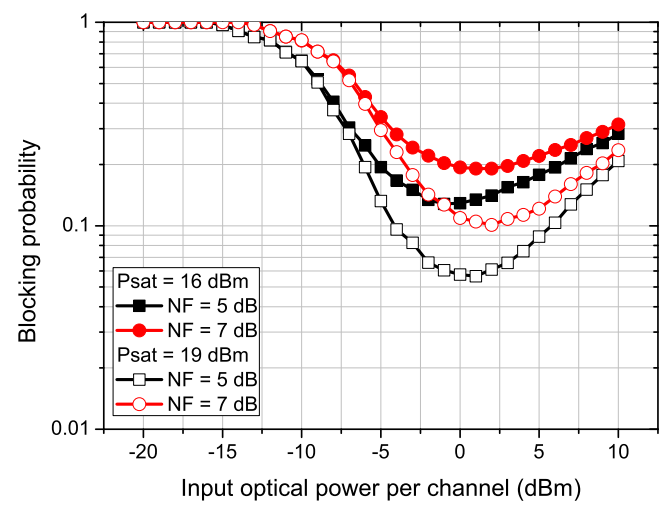

(b)

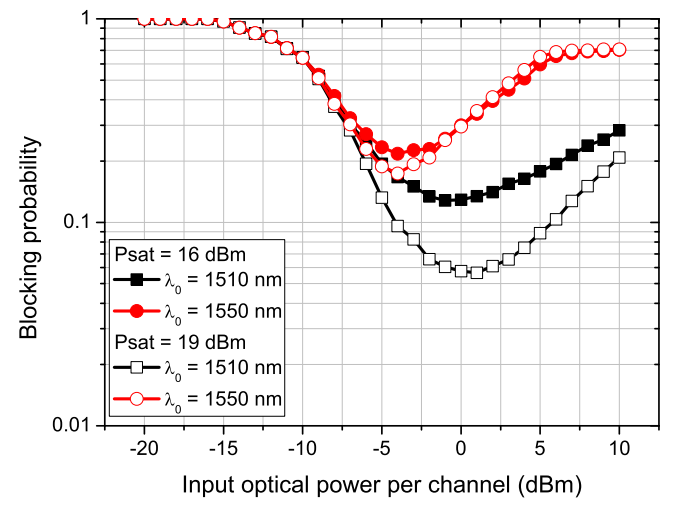

(c)

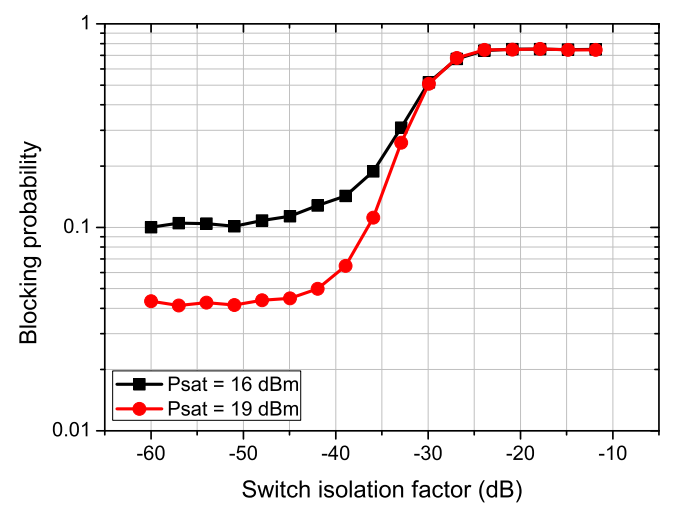

(d)

Fig. 10. Simulation example of blocking probability as a function of different: (a) network loads; (b) amplifier saturation powers and noise figures; (c) fiber type, DSF and NZ-DSF and (d) isolation factor values for optical switches. 


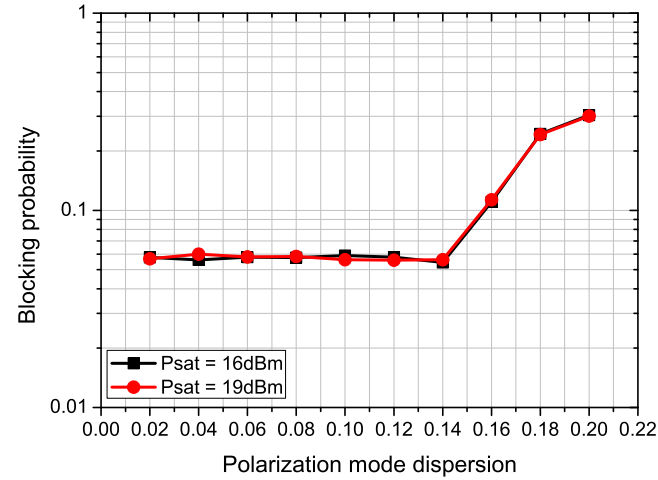

(a)

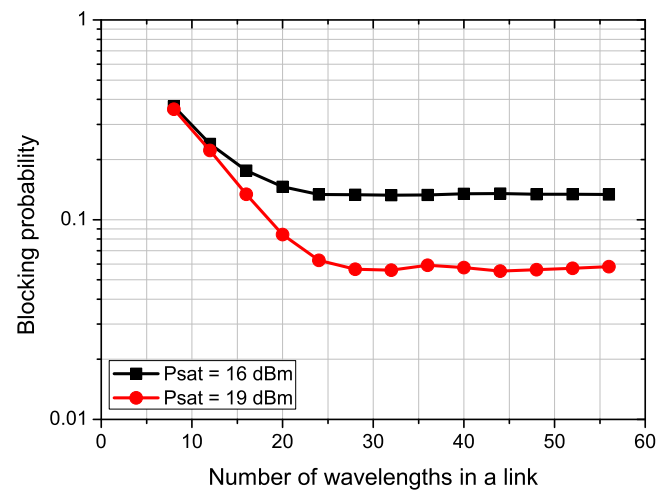

(b)

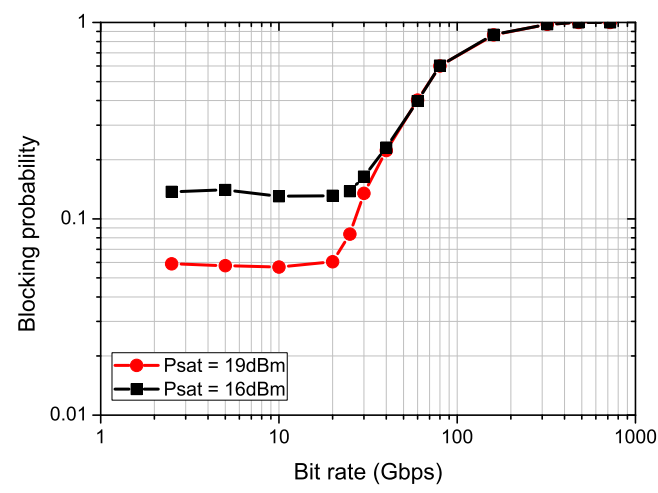

(c)

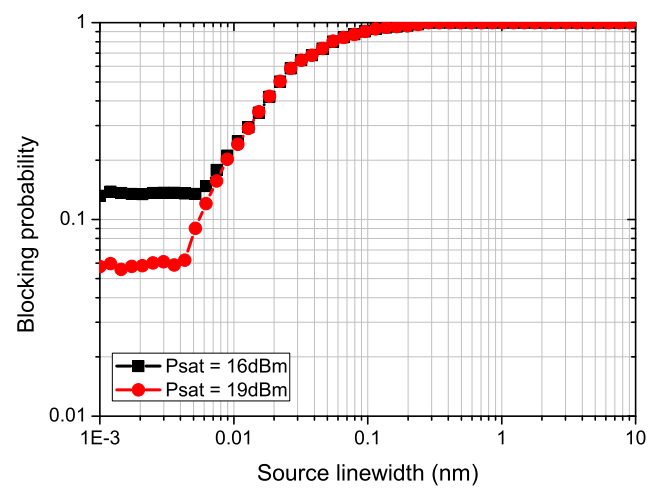

(d)

Fig. 11. Simulation example of blocking probability as a function of different: (a) PMD coefficients; (b) number of wavelengths per link; (c) transmitter bit rates and (d) transmitter linewidths.

include in future versions some other effects, such as: self-phase modulation (SPM) and cross-phase modulation (XPM) [18].

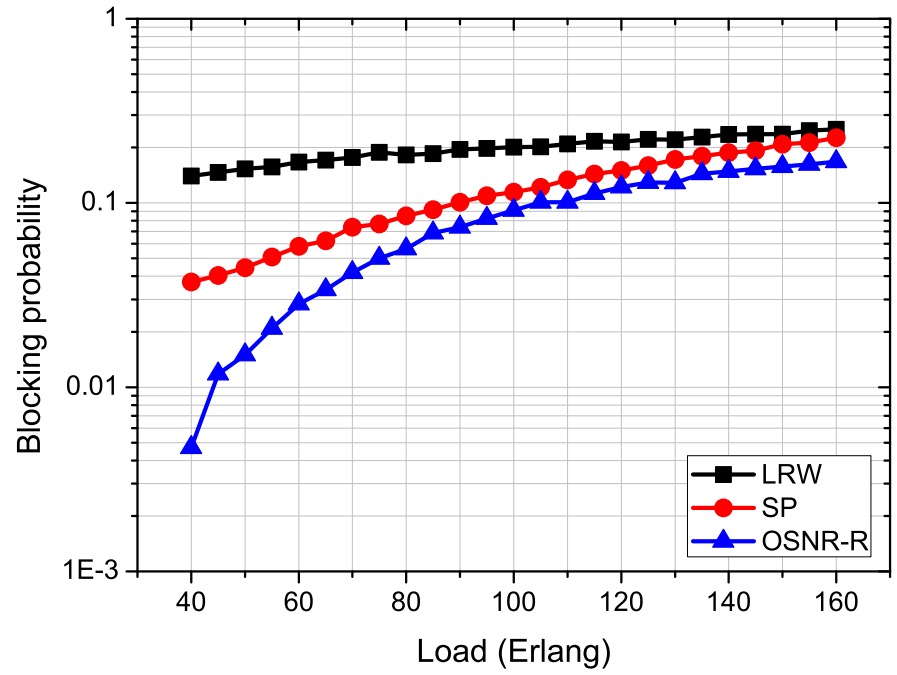

Fig. 12. Simulation example: network blocking probability as a function of network load for three different RWA algorithms (SP, LRW and OSNR-R).

The authors also intend to include some other devices such as: Raman amplifiers, wavelength converters, optical regenerators. Other network functionalities should also be included in future versions, such as: different wavelength assignment algorithms, including some impairment-aware wavelength assignment (IAWA), IA-routing (IA-R) algorithms, restoration and protection capabilities, and others.

\section{ACKNOWLEDGMENTS}

The authors acknowledge the financial support from FACEPE, CNPq, CAPES, UPE and UFPE for scholarships and grants.

\section{REFERENCES}

[1] B. Mukherjee, "Wdm optical communication networks: Progress and challenges," Journal of Selected Areas in Communications, vol. 18, no. 10, pp. 1810-1824, 2000.

[2] M. J. O’Mahony, C. Politi, D. Klonidis, R. Nejabati, and D. Simeonidou, "Future optical networks," Journal of Lightwave Technology, vol. 24, no. 12, pp. 4684-4696, December 2006.

[3] B. Ramamurthy, H. Feng, D. Datta, J. P. Heritage, and B. Mukherjee, "Transparent vs. opaque vs. translucent wavelength-routed optical networks," in Optical Fiber Communication Conference, 1999, and the International Conference on Integrated Optics and Optical Fiber Communication. OFC/IOOC., vol. 1, 1999, pp. 55-61.

[4] R. Ramaswami and K. N. Sivarajan, Optical Networks: A Practical Perspective, 2nd ed. Morgan Kaufmann, 2002.

[5] J. Strand, A. L. Chiu, and R. Tkach, "Issues for routing in the optical layer," Communications Magazine, vol. 39, no. 2, pp. 81-87, February 2001.

[6] B. Wen, N. M. Bhide, R. K. Shenai, and K. M. Sivalingam, "Optical wavelength division multiplexing (wdm) network simulator (owns): Architecture and performance studies," SPIE Optical Networks Magazine, Special Issue on Simulation, CAD, and Measurement of Optical Networks, vol. 2, 2001.

[7] "Network simulator 2," http://www.isi.edu/nsnam/ns/. Acessed in February, 2010.

[8] "Opnet simulator," http://www.opnet.com/. Acessed in February, 2010.

[9] “The nist merlin environment," http://w3.antd.nist.gov/Hsntg/prd_merlin.html. Acessed in February, 2010.

[10] "Tonets - transparent optical network simulator," http://www.nuperc.unifacs.br/grupos-de-pesquisa/grow/projetos/tonets. Acessed in February, 2010.

[11] B. Ramamurthy, D. Datta, H. Feng, J. P. Heritage, and B. Mukherjee, "Simon: a simulator for optical networks," in All-Optical Networking: Architecture, Control, and Management Issues, vol. 3843. SPIE, 1999, pp. 130-135. 
[12] D. A. R. Chaves, H. A. Pereira, C. J. A. Bastos-Filho, and J. F. Martins-Filho, "Simton: A simulator for transparent optical networks," in Proceedings of the International Telecommunications Symposium - ITS, vol. 1, Manaus, Brazil, September 2010, pp. 1-7.

[13] H. A. Pereira, D. A. R. Chaves, C. J. A. Bastos-Filho, and J. F. MartinsFilho, "OSNR model to consider physical layer impairments in transparent optical networks," Photonic Network Communications, vol. 18, no. 2, pp. 137-149, October 2009.

[14] H. A. Pereira, R. V. B. Carvalho, C. J. A. Bastos-Filho, and J. F. Martins-Filho, "Impact of amplifier noise figure modeling in simulations of impairment-aware all-optical networks," Photonic Network Communications, vol. 19, no. 1, pp. 110-120, February 2010.

[15] H. Zang, J. P. Jue, and B. Mukherjee, "A review of routing and Wavelength assignment approaches for wavelength-routed optical wdm networks," Optical Networks Magazine, vol. 1, no. 1, pp. 47-60, January 2000.

[16] S. Azodolmolky, M. Klinkowski, E. Marin, D. Careglio, J. S. Pareta, and I. Tomkos, "A survey on physical layer impairments aware routing and wavelength assignment algorithms in optical networks," Computer Networks, vol. 53, pp. 926-944, May 2009.

[17] J. He and M. Brandt-Pearce, "RWA using wavelength ordering for crosstalk limited networks," in Proceedings of the IEEE/OSA Optical Fiber Conference - OFC, Anaheim, USA, March 2006, p. OFG4.

[18] G. P. Agrawal, Fiber-Optic Communication Systems, 2nd ed. John Wiley and Sons, Inc., 1997.

[19] M. B.-P. Y. Pointurier and S. Subramaniam, "Analysis of blocking probability in noise and crosstalk impaired all-optical networks," in Proceedings of the 26th Annual IEEE Conference on Computer Communications - INFOCOM, Anchorage, USA, May 2007, pp. 2486 2490.

[20] Y. Pointurier and M. Brandt-Pearce, "Effects of crosstalk on the performance and design of all-optical networks with fiber nonlinearities," in Proceedings of the 38th IEEE Asilomar Conference on Signals, Systems and Computers, vol. 1, Monterey, USA, November 2004, pp. 83-87.

[21] N. Zulkifli, C. Okonkwo, and K. Guild, "Dispersion optimised impairment constraint based routing and wavelength assignment algorithms for alloptical networks," in Proceedings of 8th International Conference on Transparent Optical Networks - ICTON, vol. 3. IEEE/LEOS, June 2006, pp. 177-180.

[22] E. Mutafungwa and S. Halme, "Analysis of the blocking performance of hybrid ocdm-wdm transport networks," Microwave and Optical Technology Letters, vol. 34, no. 1, pp. 61-68, July 2002.

[23] B. Wen, R. Shenai, and K. Sivalingam, "Routing, wavelength and time-slot-assignment algorithms for wavelength-routed optical wdm/tdm networks," Journal of Lightwave Technology, vol. 23, no. 9, pp. 25982609, September 2005.

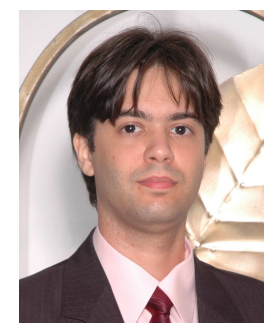

Daniel A. R. Chaves was born in Recife, Brazil, in 1981. He received the B.Sc. degree in electronics engineering from the Federal University of Pernambuco (UFPE) in 2006 and received the M.Sc. in electrical engineering in 2008 from the same institution. He is currently a Professor in the Computer Engineering Group (E-Comp) at the University of Pernambuco (UPE). Moreover, he is working toward the Ph.D. degree in electrical engineering at UFPE. $\mathrm{He}$ is currently working with the Photonics Group of UFPE, in the laboratory of optical networks. His interests are related to lightwave communication systems, including highcapacity WDM transmission, optical networking, impairment-aware routing and wavelength assignment algorithms, designing of all-optical and translucent networks, and applications of computational intelligence in optical networks optimization.

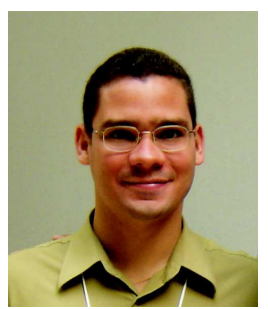

Helder A. Pereira was born in Paulista, Brazil, in 1980. He received the B.Sc. degree in Electronics Engineering from University of Pernambuco (UPE), Recife, Brazil, in 2000, the M.Sc. degree at Telecommunication National Institute (Inatel), Santa Rita do Sapucaí, Brazil, in 2002 and the $\mathrm{PhD}$ degree in Electrical Engineering at the Federal University of Pernambuco (UFPE), Recife, Brazil, in 2007. He is a professor at Polytechnic School of the University of Pernambuco (UPE).

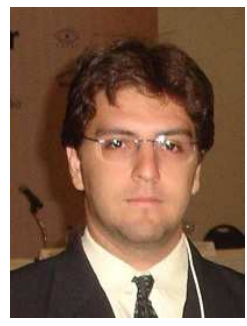

Carmelo J. A. Bastos-Filho was born in Recife, Brazil, in 1978. He received the B.Sc. degree in Electronics Engineering from Federal University of Pernambuco (UFPE) in 2000. He received the M.Sc. and $\mathrm{PhD}$ degrees in Electrical Engineering from Federal University of Pernambuco (UFPE), in 2003 and 2005 , respectively. In 2006, he received the best Brazilian Thesis award in Electrical Engineering. His interests are related to optical networks, swarm intelligence, evolutionary computation, multi-objective optimization and biomedical applications. $\mathrm{He}$ is currently an associate professor at Polytechnic School of the University of Pernambuco. He is the head of the research division of the Polytechnic School of Pernambuco. He also coordinates the Masters course in Systems Engineering.

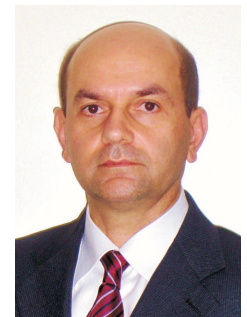

Joaquim F. Martins-Filho was born in Recife, Brazil, in 1966. He received the B.Sc. degree in Electronics Engineering from the Federal University of Pernambuco (UFPE), Recife, in 1989, and the M.Sc. degree in Physics from the same institution in 1991 , studying nonlinear optics in optical fibers. He received his $\mathrm{PhD}$ degree in Electronics Engineering from the University of Glasgow, Scotland, in 1995, investigating ulfrafast optical pulse generation from mode-locked semiconductor quantum well lasers. Since 1998 he is an associate professor in the Photonics Group, Department of Electronics and Systems of UFPE, in Recife. His current interests are in devices, subsystems, transmission systems and networking for optical communications and optical sensors.

Prof. Joaquim Martins-Filho is a Member of OSA (Optical Society of America), SBMO (Brazilian Microwave and Optoelectronics Society), SBrT (Brazilian Telecommunication Society) and SBF (Brazilian Physics Society), as well as a Research Fellow of the National Research Council of Brazil (CNPq). 